\title{
JUGENDENTWICKLUNG, GESCHLECHTSREIFE UND WECHSEL DER FÄRBUNG DES HAARKLEIDES BEIM SCHOPFGIBBON (HYLOBATES CONCOLOR LEUCOGENYS)
}

\author{
von \\ L. DITTRICH \\ Zoologischer Garten Hannover, Adenauerallee 3, D-3000 Hannover, B.R. Deutschland
}

\section{ZUSAMMENFASSUNG}

Beschreibung des Verlaufes der Umfärbung eines Schopfgibbon-Weibchens beim Wechsel des Jugend- zum Alterskleid, beginnend im Alter von $71 / 2$, ausgefärbt mit $81 / 2$ Jahren. Die Geburt des ersten Kindes erfolgte, als das Weibchen eben 9 Jahre alt geworden war, des zweiten $21 / 2$ Jahre später. Auch der Verlauf der Umfärbung des erstgeborenen weiblichen Kindes beim Wechsel vom Kleinstkind- zum Jugendkleid, beginnend als es 9 Monate, abgeschlossen als es $11 / 2$ Jahre alt war, sowie sein allmähliches Selbständigwerden vom 6./7. Monat an bis zum Alter von etwa 2 Jahren, werden beschrieben.

\section{ABSTRACT}

Description of the process of colour change in the fur of a female White-cheeked Gibbon from the juvenile black coat to the light adult coat, starting at the age of $71 / 2$ and finishing at $81 / 2$ years. The birth of the first young occurred when the female had attained the age of barely 9 years, and of the second $21 / 2$ years later. The progress of colour change of the first-born female young from the light infant fur to the dark juvenile fur, starting at the age of 9 months and finishing at the age of $11 / 2$ years, is described as well as its gradual growth of independence from the 6 th/ 7 th month up to the age of about 2 years.

Anfang Oktober 1969 erwarb der Zoologische Garten Hannover aus Laos 1,1 Schopfgibbons, Hylobates (Nomascus) concolor leucogenys Ogilby, 1840. Die beiden noch jugendlichen Tiere trugen ein schwarzes Haarkleid bis auf den weißen Backenbart, der sowohl für jugendliche Tiere beiderlei Geschlechts als auch für die adulten Männchen dieser Unterart charakteristisch ist. Sie waren gleichgroß und hatten etwa $2 / 3$ der Körpergröße eines erwachsenen Schopfgibbons erreicht. Nach Auskunft des Lieferanten sollten sie etwa $21 / 2$ Jahre alt sein, eine Angabe, die sowohl ihrem Entwicklungszustand $z u$ entsprechen schien und mit dem eines späteren Kindes dieses Paares im selben Alter übereinstimmte, als auch den Angaben von Ellefson (1974) über das Alter vorzugsweise gefangener bzw. in Menschenhand gelangender junger Gibbons entsprach. Junge Gibbons sollen dann, wenn sie eben von ihren Eltern etwas unabhängiger geworden sind, also $21 / 2$ jährig, am ehesten in die Hand der Fänger geraten. Bis zum Alter von mindestens 2 Jahren werden Gibbonkinder in kritischen Situationen noch von der Mutter getragen (Ogilvie, 1923).

Im Dezember 1974, etwa 71/2 Jahre alt, begann sich der weibliche Schopfgibbon umzufärben, indessen das Männchen erwartungsgemäß die juvenile Fellfärbung behielt. Zunächst wurde das bisher schwarze Haarkleid des Weibchens mehr und mehr aschgrau, vor allem an den Kopfseiten, am Hinterkopf, im Bereich der Schultern und der Schlüsselbeine, der Brust sowie an den Oberarmen und -schenkeln. Innerhalb eines Vierteljahres schritt die Aufhellung stetig voran, so da $\beta$ am Oberkopf nunmehr der dunkle Fellstreifen deutlich hervortrat und sich markierte, der beim adulten Weibchen sich von der Stirn bis zum Hinterkopf entlangzieht. Noch blieb der juvenile weiße Bart im Gesicht auffällig sichtbar. Zugleich erschien jetzt das Gibbonweibchen ein wenig größer und kräftiger als der gleichaltrige männliche Partner.

Innerhalb des nächsten Vierteljahres, also bis zur Vollendung des 8. Lebensjahres, waren Brust, Schlüsselbeinregion, Schultern, Oberarme und -schenkel des Weibchens hellgrau. Das schwarze Kopfband trat deutlich hervor und der weiße Backenbart ging mehr und mehr in der immer heller werdenden Färbung der Kopfhaare unter. Nur Füße und Hände waren, vor allem wohl der 
dort schütteren Behaarung wegen, noch sehr dunkel. Das Fell des übrigen Körpers erschien nun hell-aschgrau.

Im darauffolgenden dritten Vierteljahr nach Beginn der Umfärbung wurde das Haarkleid des Tieres vollends hell. Nur die Füße und Hände, Unterschenkel und -arme waren noch dunkelgrau. Als das Gibbonweibchen schließlich gegen Ende des Jahres 1975 ein Alter von etwa 81/2 Jahren erreicht hatte, war es bis auf das typische schwarze Kopfband und einige schwarze Haare an den Ohrmuscheln vollständig hell geworden. Die Umfärbung vom Jugend- zum Alterskleid hatte also genau ein Jahr gedauert. Der Verlauf des Farbwechsels der Behaarung stimmt im wesentlichen mit den Angaben von Pocock (1905) überein, doch konnte damals eine Altersbestimmung des als $H$. c. hainanus beschriebenen weiblichen Schopfgibbons nicht vorgenommen werden.

Von Ende Oktober $1975 \mathrm{ab}$, als die beiden Schopfgibbons also etwa $81 / 2$ Jahre alt waren, kam es zwischen ihnen zu sexuellem Verhalten. Das Weibchen legte sich öfter bäuchlings auf den Boden nieder und streckte dem Männchen die Anal-Genitalregion leicht angehoben entgegen. Das Männchen rutschte, von hinten kommend, an das Weibchen heran. Die Kopulationsdauer war stets sehr kurz, meist kürzer als $1 / 2$ Minute. Durch die Vielzahl der Paarungen konnte die schließlich die Trächtigkeit bewirkt habende nicht ermittelt werden, zumal des Pärchen noch kopulierte, als das Weibchen schon sichtbar trächtig war.

Über den Eintritt der Geschlechtsreife und damit den Abschluß der Umfärbung bei den farbdimorphen Hylobates-Arten $H$. concolor, $H$. hoolock und $H$. pileatus gehen die Angaben in der Literatur beträchtlich auseinander. Nach Dandelot (1960) sollen die Hylobates-Arten im Alter von 5-6 Jahren, nach Ellefson (1974) Lars 6-jährig, Hulocks nach McCann (1933) und Peart (1934) mit 5 oder 6 Jahren und schließlich Schopf gibbons nach Delacour (1951) und Marshall et al. (1972) wenn sie 5-8 Jahre alt sind, geschlechtsreif werden. Diesen Angaben liegen aber nur Schätzungen des Alters der Gibbons zugrunde. Auch die Altersangabe von Schultz (1944) eines Gibbons nicht genannter Art, der erst im Alter von 8 Jahren und
10 Monaten geschlechtsreif geworden sein soll, beruht auf einer Schätzung. Lafore (zit. nach Groves, 1972) erhielt ein in der juvenilen Umfärbung begriffenes, also ca. 11/4 Jahre altes Schopfgibbonweibchen, bei dem sich allerdings schon im Alter von $41 / 4$ Jahren ganz langsam das Jugendkleid auszubilden begann. Als der Gibbon $63 / 4$ Jahre alt war, hatte er sich völlig umgefärbt. Nach Poglayen-Neuwall (1977) war ein im Alter von 2 Monaten übernommenes und künstlich aufgezogenes Larweibchen 9 Jahre alt, als es sein erstes Kind brachte. Das Alter des Vaters wurde auf nur 6 Jahre geschätzt; die Tragzeit hatte 205 Tage gedauert.

In der neueren Literatur wird die Tragzeit der Hylobates-Arten mit etwa 7 Monaten veranschlagt, z.B. von Schultz (1956), bzw. sogar mit nur 199 Tagen angegeben (Voss, 1970). Für den Schopfgibbon gab Rode (1942) 200-212 Tage als Tragzeitlänge an.

$\mathrm{Da}$ die Geburt unseres Schopfgibbonkindes bereits Anfang Mai 1976 erfolgte, mußte schon eine der Paarungen, die zu Beginn der geschlechtlichen Aktivität lag, noch in der Zeit der Umfärbung des Weibchens, zur Trächtigkeit geführt haben. Anzeichen für Trächtigkeit - Anschwellen des Leibes - wurden erstmals im März, d.h. am Ende des 4. Monats der Schwangerschaft bemerkt. Die Geburt erfolgte in der Nacht vom 4. zum 5. Mai 1976, als nach unserer Schätzung das Weibchen eben 9 Jahre alt geworden war. Nach Haggard (1965) war ein im Tiergarten geborenes Larweibchen bei der Geburt seines ersten Kindes 9 Jahre und 11 Monate alt.

Die Mutter zog ihr Kind, ohne daß Schwierigkeiten aufgetreten wären, groß. Zunächst war auch der Schopfgibbon-Neonatus, wie die Neugeborenen der anderen $H$ ylobates-Arten, fast nackt und nur von ganz feinen, fast farblosen hellen Haaren, die recht schütter standen, bedeckt. Nur die Kopfplatte wies eine etwas dichtere Behaarung auf. Noch im Laufe des ersten Lebensmonats bildete sich aber vor allem auf dem Rücken und an den Oberarmen und -schenkeln ein hellgelbliches, etwas längeres Haarkleid aus. Der Bauch blieb zunächst noch nackt und war bei dem vierteljährigen Baby so schütter behaart, daß überall die 

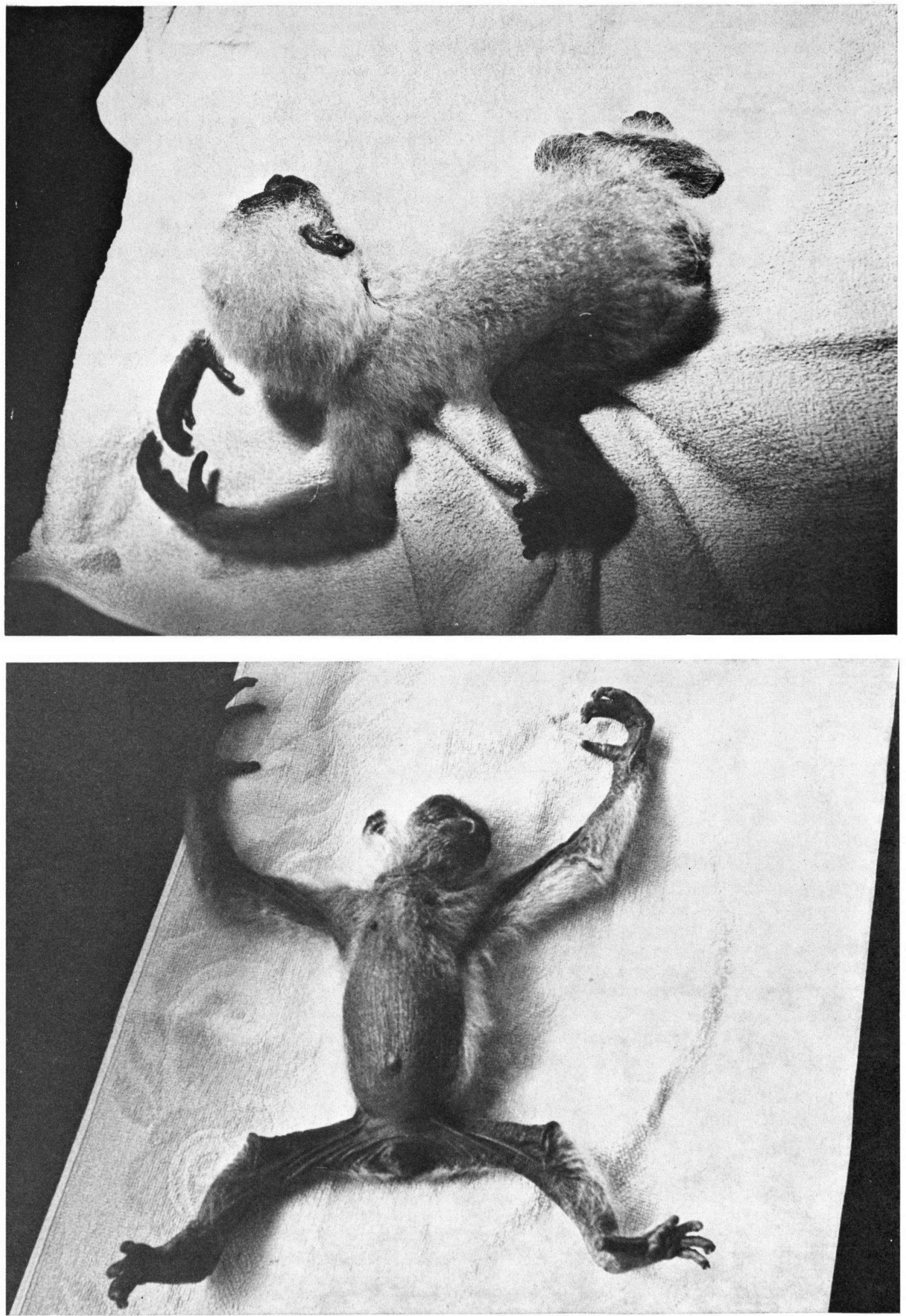

Abb. 1 (a \& b). Sechs Wochen altes männliches Schopfgibbonkind, auf Kopf und Rücken gut behaart, am Bauch und an den Innenseiten der Oberarme bzw. -schenkel, die dem mütterlichen Körper anliegen, fast nackt (Aufnahmen: U. Rottmann). 
Körperhaut durchschimmerte. Auch die Handrücken, der mediale Teil der Unterarme und die Fußrücken waren so spärlich behaart, daß die Haut durchschimmerte, doch da diese sich rasch dunkel pigmentierte, erschienen diese Partien dunkel im sonst rein hellgelblich-weißen Fell des Kindes.

Auch über die Umfärbung des Babykleides zu dem des jugendlichen Gibbonkindes weichen die Angaben in der Literatur erheblich voneinander ab. Nach Marshall et al: (1972) färben Gibbonkinder im Alter von „a few months" um. Schopfgibbonkinder sollen nach Napier \& Napier (1967) im Alter von 6 Monaten das schwarze juvenile Fell anlegen und Larkinder färben nach Groves (1972) im Alter von 6-9 Monaten um, doch sollen sie schon älter als $1 \mathrm{Jahr}$ alt sein, ehe sich das dunkle Jugendkleid vollständig ausgebildet hat. Fooden (1969) zitiert Berkson (mündl. Mitt.), nach dem Kappengibbonkinder das helle Baby-Haarkleid bis zum Alter von 2-3 Jahren tragen sollen. Wiederum scheinen die meisten Angaben über das Alter der die Fellfarbe wechselnden Junggibbons nur auf Schätzungen zu beruhen.

Bei unserem Schopfgibbonkind, einem Weibchen, zog sich der Ersatz des hellen Babyhaarkleides durch das schwarze Jugendkleid über einen Zeitraum von 9 Monaten hin. Als das Gibbonkind $4 \frac{1}{2}$ Monate alt geworden war, fielen im Laufe der nächsten $11 / 2$ Monate aus dem Fell des Hinterkopfes mehr und mehr Haare aus, so daß dort schließlich die dunkle Haut hindurchschimmerte. Halbjährig war es noch gänzlich von hellem Fell bedeckt und hatte eine Größe wie ein mütterlicher Oberschenkel. Im 7. und 8. Monat erfuhr das Baby einen sichtbaren Entwicklungsschub, zeigte aber noch keinerlei Anzeichen für einen Fellwechsel.

Erst zu Beginn des 9. Lebensmonates und ganz allmählich bis zum Ende des 10. Lebensmonates zunehmend, konnten bei einer Betrachtung des Kindes aus nächster Nähe, dort, wo ein halbes Jahr zuvor der Haarausfall aufgetreten war und wo sich später beim adulten weiblichen Schopfgibbon das dunkle Band über den Kopf bis zum Nacken zieht, mehr und mehr dunkle Haare bemerkt werden. Mitte des 11. Lebensmonats war diese dunklere Fellpartie auf dem Kopf auch von fern zu erkennen. Ende des 11. Lebensmonats erschien ein schmaler Streifen, der sich, dem Verlauf der Wirbelsäule folgend, vom Nacken bis in die Nierengegend erstreckte, etwas dunkler gefärbt als die übrigen Partien des Rückenfelles. Vom Beginn des 12. Lebensmonats an werden Rücken, Unterarm und -schenkel mehr und mehr grau, so daß das Jungtier einjährig zwar noch völlig hell erscheint, aber nicht mehr so weißlichgelb, sondern hellaschgrau gefärbt. Kopfband, Rückenmitte, Ellbögen, Unterarm und -schenkel sind etwas dunkler als der übrige Körper.

Im Verlaufe des 13. und 14. Lebensmonats wurde das Gibbonkind zunehmend dunkler, grau, aschgrau, schließlich dunkelgrau. Es hatte zu Beginn des 15 . Lebensmonats eine Körperlänge erreicht, die der Länge des Beines der Mutter entspricht. Im Verlaufe des 15. Monats vertiefte sich die dunkle Farbe so, daß das Kind insgesamt als dunkelgrau, wenn auch noch nicht schwarz erschien. Schon in der zweiten Hälfte des 14. Lebensmonats und verstärkt im 15. Monat dunkelte das Fell des Kopfes derart, daß die hell bleibenden Partien, die den weißlichen Backenbart des juvenilen Tieres bilden, mehr und mehr hervortreten. Allerdings waren Stirn und Kopfseiten noch so hell, daß die dunklere Mittelpartie im Bereiche des dunklen Kopfbandes beim adulten weiblichen Schopfgibbon noch als dunklere Zone auffiel. Auch die Brust und der Oberbauch waren noch heller als der übrige Körper. Der Handrücken war schon tiefschwarz.

Im Laufe des 15. und in der ersten Hälfte des 16. Lebensmonats vertiefte sich die Fellfarbe des Gibbonkindes schrittweise zum Schwarzgrau. Die mittlere Region des Oberkopfes war stets dunkler als die Umgebung. Mehr und mehr trat der helle Backenbart im Gesicht hervor. Etwas heller als der übrige Körper waren Unterarm, Unterschenkel und die Kopfseiten. Vom Ende des 16. Lebensmonats an und im Verlaufe des 17. wird das Fellkleid des Gibbonkindes allmählich schwärzlich, erreicht allerdings noch nicht die tiefschwarze Färbung des adulten Männchens. Noch immer sind die Kopfseiten heller gefärbt, oberhalb der Augenregion erkennt man je einen unregelmäßig begrenzten hellen Fleck mit einem Durchmesser von etwa $1 \mathrm{~cm}$. Auch die Behaarung der Brustwarzen ist heller.

Im Verlaufe des 18. Lebensmonats erreicht die 
Farbe des Haarkleides, bis auf die grauen „Uberaugenflecke" und die hellen Haare im Bereich der Brustwarzen und natürlich bis auf den leuchtend hellen Backenbart, die tiefschwarze Tönung des Fellkleides des Vaters. Die Umfärbung des juvenilen Gibbons ist damit abgeschlossen. Sie begann ganz allmählich, als das Gibbonkind 9 Monate alt war, veränderte vor allem in seinem 14. und 15. Monat das helle Baby-zu einem grauen úbergangskleid und fand ihren Abschluß 9 Monate später, als das Gibbonkind gerade $11 / 2$ Jahre alt geworden war. Der junge Gibbon hat stehend nun die Größe des Rumpfes der Mutter (also ohne deren Kopf und Beine) erreicht.

Einige Beobachtungen sollen das Selbständigwerden des Kindes, das sich auf die Zeit vom 4. bis zum 17./18. Lebensmonat erstreckte, verdeutlichen. Im ersten Vierteljahr nahm das stets am Bauch der Mutter hängende Kind nicht merkbar

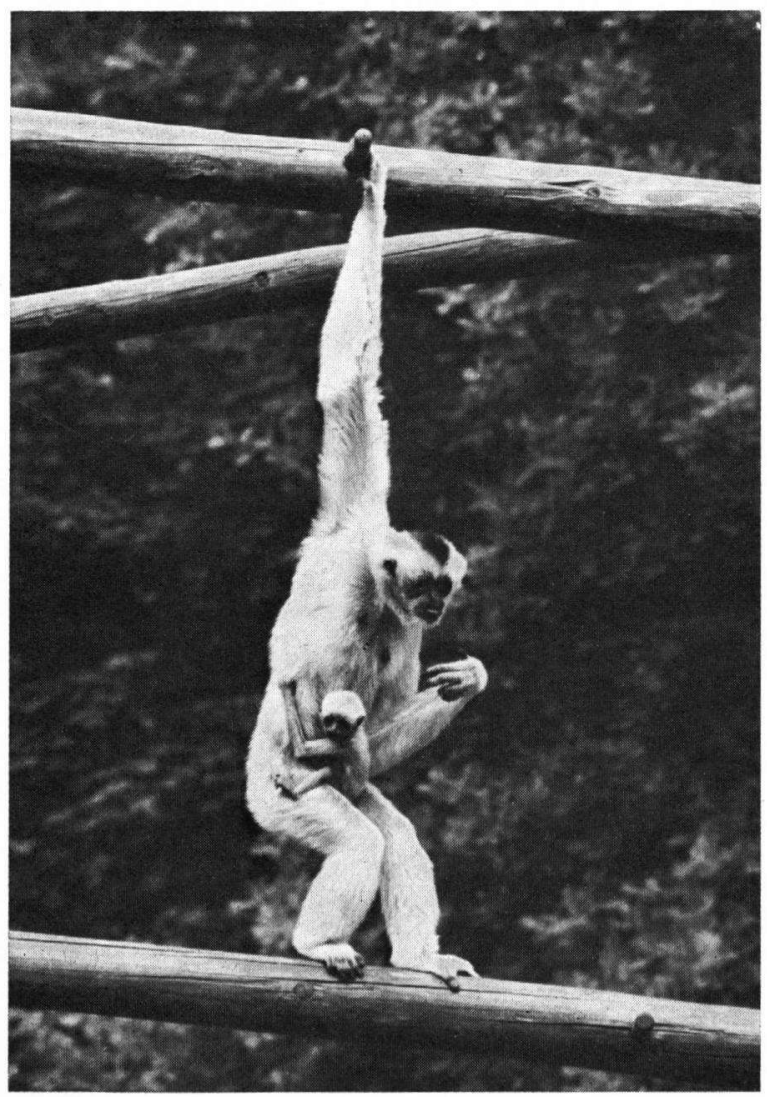

Abb. 2. Schopfgibbonweibchen mit 3 Wochen altem Kind (Aufnahme: U. Brandt).
Anteil an den Vorgängen in seiner Umwelt, auffällig etwa durch Kopf- oder Körperbewegung. Am Ende des 3. Monats mußte das Baby seine Verankerung mit den Fingern im Fell der Mutter etwas lösen. Es war auffällig, daß diese ihr Kind häufig mit der unter dem Körper gehaltenen Hand stützte, wenn sie aufstand, was früher nicht zu sehen war. Mit den Zehen krallte sich das Gibbonkind niemals, weder früher noch später, im Fell der Mutter fest. Vielmehr preßte es Schenkel und Füße an den Körper und sicherte sich auf diese Weise. Die Zehen waren, ohne Haare der Mutter zu fassen, eingekrümmt. Am Ende des 3. Lebensmonats wurden leere Kaubewegungen bei dem Affenkind beobachtet, wenn die Mutter fraß.

Im Verlauf des 4. Monats begann das Schopfgibbonkind immer häufiger, aber offenbar stets erfolglos, nach Futterbrocken zu greifen, die sich die Mutter zum Munde führte. Es kratzte sich, an der Mutter hängend, mit einem Hinterbein am Körper. Ende des 4. Lebensmonats saß es wiederholt neben der am Boden ruhenden Mutter, durch beide Hände fest mit ihr verankert.

Im Laufe des 5. Monats hing das Kind nach wie vor fest am Körper der Mutter. Als diese sich eines Tages mit dem Männchen am Boden balgte, wurde das Kind durch die auf dem Bauch liegende Mutter fest auf den Boden aufgepreßt. Ein Klagelaut war nicht zu hören. Wenn die Mutter ruhte, krabbelte das Kind in ihrem Schoß umher, ohne sich mit den Händen zu lösen. Dabei versuchte es auch, sich auf die Beine zu stellen, was nicht gelang, dabei stets sofort mit mindestens einer Hand wieder in das Fell der Mutter greifend.

Im Verlauf des 6. und 7. Monats gewann man den Eindruck, daß das Kind von der Mutter freikommen wollte. Es griff nach Futterbrocken, die in Reichweite lagen, oder ins Klettergerüst. Die Mutter verhinderte alle diese Bemühungen. Erst am Ende des 7. Monats wurde es freisitzend neben der Mutter beobachtet, und für einen kurzen Moment stellte es sich mit hochgehobenen Armen aufrecht auf die Beine.

Die Temperaturen waren in diesem Novemberund Dezembertagen niedrig, oft war es sehr feucht, es regnete viel. Vielleicht spielte das Wetter eine Rolle für die beträchtliche Entwicklungsverzögerung, die das Schopfgibbonkind zeigte im Ver- 
gleich zu den Angaben in der Literatur über die frühe Entwicklung von Larkindern (vgl. Berkson, 1966, Brody \& Brody, 1974, Crandall, 1964 und Meyer-Holzapfel, 1950). Es muß aber auch daran gedacht werden, daß sich Larkinder vom 2. Monat an schneller entwickeln, sowohl was Futteraufnahme, das Lösen von der Mutter, selbständiges Sitzen, Stehen und vor allem aber Hangeln und Klettern anlangt. Im zweiten Jahr haben sie den Angaben der Literatur zufolge einen mehrmonatigen Entwicklungsvorsprung vor unserem Schopfgibbonkind.

$\mathrm{Zu}$ Beginn des 8. Lebensmonats, 4 bis 5 Monate später als vom Lar beschrieben, hing das Schopfgibbonkind erstmals frei am Gitter des Innenkäfigs. Die Mutter war in unmittelbarer Nähe und nahm nach wenigen Augenblicken das sich nicht bewegende Kind wieder an den Körper. Erst im Verlaufe des 10. Monats löste sich das Kind völlig und bald immer häufiger von der am Boden liegenden Mutter und zeigte die bekannten „Froschsprünge" der Gibbonkinder. Am Körper der Mutter begann es umherzuklettern. Es stieg auf ein neben der Muttter ruhendes Klammeraffenweibchen, das seinerseits einen Säugling am Körper trug, und kletterte auch auf ihm umher. Sowie die Mutter aber durch Aufrichten des Körpers deutlich werden ließ, daß sie aufstehen und weggehen wollte, strebte das Kind zu ihr zurück.

Erst am Ende des 12. und 13. Lebensmonats löste es sich am Boden soweit von der Mutter, $\mathrm{da} ß$ es allein zurückblieb, wenn diese sich in der Nähe bewegte. Auch der Vater nahm jetzt das Kind und trug es kurze Zeit. Offenbar ,sah" die Mutter dies nicht gern, denn stets kam sie bald herbei, und das Kind stieg auf sie über. Als die Mutter einmal aus dem Blickfeld des Kindes verschwand und in den Innenstall ging, begann das Kind sofort laut zu schreien, worauf sie augenblicklich zu ihm zurückkehrte und es wieder aufnahm. Im Beisein der Mutter hüpfte das Kind häufig froschartig auf dem Boden umher, suchte Futterbrocken und fraß davon. Obwohl es, nach dem Zustand der Zitzen der Mutter zu urteilen, noch ausgiebig saugen mußte, was nicht beobachtet werden konnte, fraß es jetzt reichlich mit vom Futter der Eltern. Immer noch nahm die Mutter allerdings dem Kind Futterbrocken weg, um sie selbst aufzufressen.

Erst am Ende des 15. Monats begann das auf

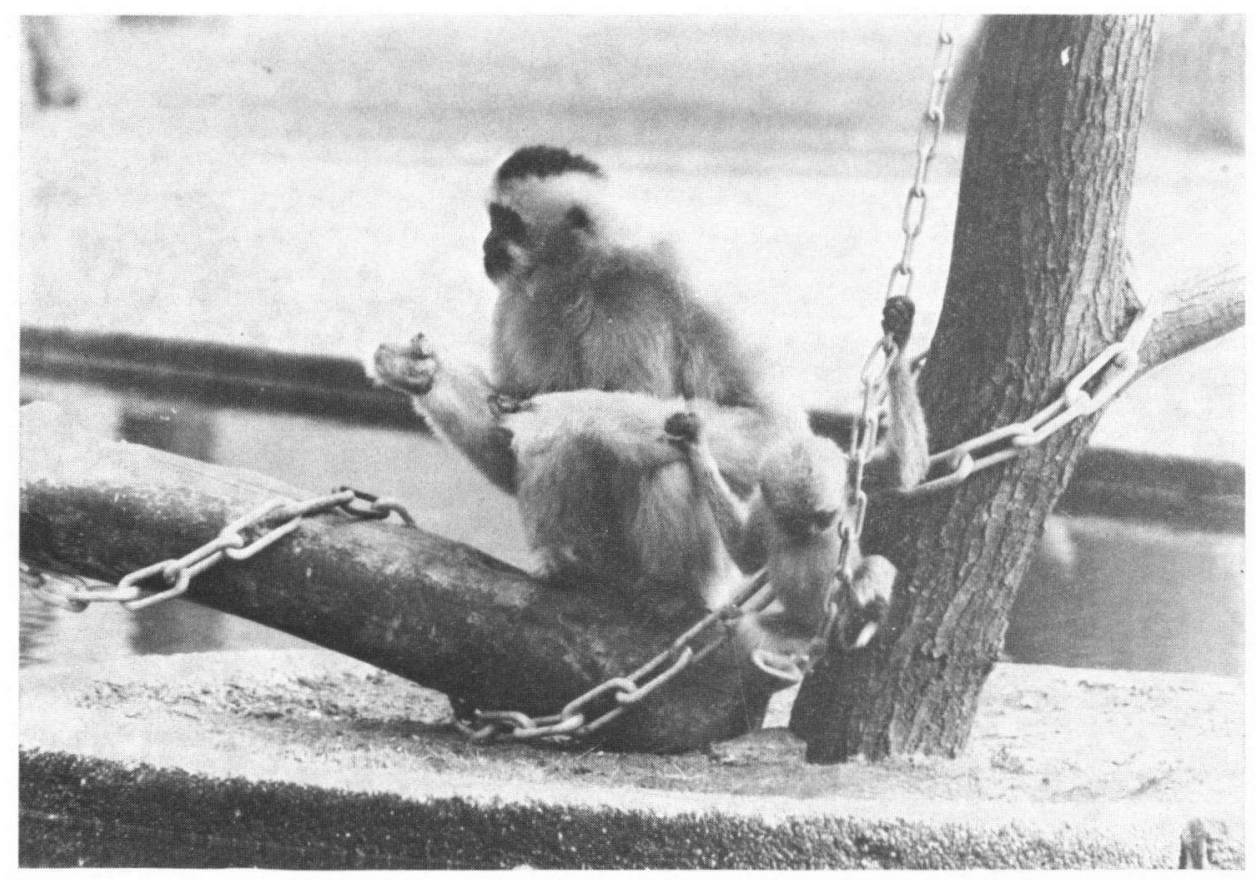

Abb. 3. Sechs Monate altes Schopfgibbonkind, die Umwelt explorierend, dabei aber in Hautkontakt mit der Mutter bleibend (Aufnahme: U. Brandt). 
dem Boden sehr beweglich gewordene und auf allen vieren schnell laufende Kind am Klettergerüst zu klettern, weiträumige Hangelschwünge waren noch nicht zu sehen. Das Kind kletterte an einer Kette hoch hinauf ins Klettergerüst und sprang aus einer Höhe von etwa $1 \mathrm{~m}$ zum Boden herab. Immer noch wurde es die meiste Zeit über von der Mutter getragen, wobei es oft geradezu „lässig" am Körper der Mutter hing, z.B. nur mit einer Hand in ihrem Fell verankert und die Schenkel angepreßt, in der anderen Hand aber einen Futterbrocken haltend, von dem es, während die Mutter sich bewegte, abbiß. Im Klettergerüst vermochte es noch nicht sich sicher zu halten. Einige Male stürzte es zu Boden, wenn die Eltern oder die mit den Schopfgibbons die Anlage bewohnenden Klammeraffen die Kletterstangen in starke Schwingungen versetzten. Einmal fiel es in das etwa $35 \mathrm{~cm}$ tiefe die Anlage umgebende Wasserbecken und verschwand unter der Wasseroberfläche. Die Mutter kam blitzschnell herbei, griff ins Wasser und zog ihr Kind wieder heraus. Nach diesem Erlebnis löste sich das Kind den ganzen Tag über nicht mehr von der Mutter.

Im Laufe des 17. Monats kletterte und hangelte das Gibbonkind selbständig weitab von der Mutter im Klettergerüst. Die Fähigkeit, größere Strecken hangelnd zu überwinden, nahm rasch zu. Nicht nur auf dem Boden, auch hoch oben auf den armstarken Kletterstangen richtete es sich jetzt auf den Hinterbeinen auf und lief biped einige noch etwas unbeholfen wirkende Schritte.

Im Verlauf des 18. Monats vermochte es gut, wenn auch noch nicht so ausdauernd wie die Eltern, aufrecht zu gehen. Obwohl es immer noch sehr viel getragen wurde, spielte und kletterte es immer häufiger weitab von der Mutter im Gerüst der Außenanlage.

Gegen Ende seines zweiten Lebensjahres wurde der junge Gibbon nur noch selten von der Mutter getragen, aber immer noch regelmäßig gesäugt. Im Juli 1978, als er 26 Monate alt geworden war, versiegte die Brust der Gibbonmutter. Nachts schlief das Jungtier noch eng an die Mutter geschmiegt. Erst wenige Tage vor der Geburt des nächsten Kindes mußte es sich einen eigenen Schlafplatz getrennt von dem der Mutter im Innenkäfig suchen.
Schon vom Sommer 1977 an, als das Gibbonkind 1 Jahr alt geworden war, paarten sich die Eltern regelmäßig. Am 26. November 1978 gegen 20.00 wurde ihr nächstes Kind geboren (ein Männchen), so daß die Paarungen in der Zeit vom 25-29. April zur Trächtigkeit geführt haben mußten.

\section{LITERATUR}

ARNOLD, R. C., 1973. Births of gibbons in captivity. In: D. M. Rumbaugh ed., Gibbon and siamang, 2: 221-227 (Karger, Basel).

Badham, M., 1967. A note on breeding the Pileated Gibbon Hylobates lar pileatus at Twycross Zoo. Int. Zoo Yb., 7: 92-93.

BerksoN, G., 1966. Development of an infant in a captive gibbon group. J. Genet. Psychol., 108 (2): 311-325.

Berkson, G. \& V. Chaicumpa, 1969. Breeding gibbons (Hylobates lar entelloides) in the laboratory. Lab. Animals Care, 19: 808-811.

Brody, E. J. \& A. E. BRODY, 1974. Breeding Müller's Bornean Gibbon Hylobates lar muelleri. Int. Zoo Yb., 14: 110-113.

Carpenter, C. R., 1940. A field study in Siam of the behavior and social relations of the gibbon (Hylobates lar). Comp. psychol. Monogr., 16 (5): 1-212.

,- 1941 . The menstrual cycle and body temperature in two gibbons (Hylobates lar). Anat. Rec., 79: 291-296.

- 1958 . Soziologie und Verhalten freilebender nichtmenschlicher Primaten. Handb. Zool., Berl., 8 (18), 10. Teil (II): 1-32.

Coolidge, H. J., 1933. Notes on a family of breeding gibbons. Hum. Biol., 5: 289-294.

Crandall, L. S., 1964. The management of wild mammals in captivity: i-xv, 1.761 (Univ. Chicago Press, Chicago \& London).

Dandelot, P., 1960. Les Gibbons, gracieux et merveilleux acrobates. Sci. Nat., Paris, 39: 2-11.

Delacour, J., 1951. La systématique des Gibbons indochinois. Mammalia, 15: 118-123.

-, 1961. Gibbons at liberty. Zool. Gart. Lpz., (N.F.) 26 (1/2): 96-99.

Ellefson, J. O., 1974. A natural history of White-handed Gibbons in the Malayan Peninsula. In: D. M. Rumbaugh ed., Gibbon and siamang, 3: 1-136 (Karger, Basel).

FoODEN, J., 1969. Color-phase in gibbons. Evolution, 23 (4): 627-644.

$\longrightarrow, 1971$. Color and sex in gibbons. Bull. Field Mus. nat. Hist., 42 (6) : 2-7.

Groves, C. P., 1972. Systematics and phylogeny of gibbons. In: D. M. RumbAugh ed., Gibbon and siamang, 1: 1-89 (Karger, Basel).

Haggard, V. D., 1965. Lar Gibbon Hylobates lar breeding records. Int. Zoo Yb., 5: 110-111.

Hill, C. A., 1967. A note on the gestation period of the siamang Hylobates syndactylus. Int. Zoo Yb., 7: 93-94.

Hinl, W. C. O., 1949. Gibbons. Zoo Life, 4 (2): 40-42.

Hutzelsider, H. B., 1937. Bericht über eine Gibbon-Geburt im Zoologischen Garten von Aarhus (Dänemark). Zool. Gart. Lpz., (N.F.) 9 (3/4): 113-122. 
IBSCHER, L., 1967. Geburt und frühe Entwicklung zweier Gibbons (Hylobates lar L.). Folia primatol., 5: 43-69.

KeEling, M. E. \& H. M. MCCluRe, 1972. Clinical management, diseases and pathology of the gibbon and siamang. In: D. M. Rumbaugh ed., Gibbon and siamang, 1: 207-249.

LANCASTER, W. E., 1966. A further note on the breeding records of Lar Gibbons, Hylobates lar, at Adelaide Zoo. Int. Zoo Yb., 6: 147.

Marshall, J. T. jr., D. A. Ross \& S. Chantharojvong, 1972. The species of gibbons in Thailand. J. Mammal., 53: $479-486$.

MCCANN, C., 1933. Notes on the colouration and habits of the White-browed Gibbon or Hoolock (Hylobates hoolock Harl.). J. Bombay nat. Hist. Soc., 36: 395-405.

MeYer-Holzapfel, M., 1950. Beobachtungen über das Verhalten einer Gibbonfamilie im Zürcher Zoologischen Garten. Zool. Gart. Lpz., (N.F.) 17 (1/5): 10-27.

NAPIER, J. R. \& P. H. NAPIER, 1967. A handbook of living Primates. Morphology, ecology and behaviour of nonhuman Primates: i-vii, 1-456 (Academic Press, London/ New York).

OgILviE, A. W., 1923. Breeding of the gibbons in captivity. J. nat. Hist. Soc. Siam, 6 (1): 137.

PearT, J. F., 1934. Notes on the colouration of the Whitebrowed Gibbon (H. hoolock Harl.). J. Bombay nat. Hist. Soc., 37: 214.

Pocock, R. I., 1905. Observations upon a female specimen of the Hainan Gibbon (Hylobates hainanus), now living in the Society's gardens. Proc. zool. Soc. Lond., 1905 (2): 169-180, pl. V.

Poglayen-Neuwall, I., 1977. Parturition in a hand-reared, primiparous gibbon (Hylobates lar). Zool. Gart. Jena, (N.F.) 47 (1): 57-58.
RIESS, B. F., 1956. The behavior and social relations of the gibbon (Hylobates lar) observed under restricted freerange conditions. Zoologica, N.Y., 41: 89-99.

Robinson, S. M., 1925. Birth of a White-handed Gibbon (Hylobates lar) in captivity. J. Bombay nat. Hist. Soc., 30: $456-458$.

Rode, P., 1942. A propos de la durée de gestation chez les Gibbons. Mammalia, 6: 46-47.

Rumbaugh, D. M., 1965. The gibbon infant, Gabrielle: its growth and development. Zoonooz, 38 (12): 10-15.

- 1967. The siamang infant, Sarah... its growth and development. Zoonooz, 40 (3): 12-18.

SASAKI, T., 1962. Hand-rearing a baby gibbon (Hylobates lar). Int. Zoo Yb., 4: 289-290.

Schulrz, A. H., 1944. Age changes and variability in gibbons. A morphological study on a population sample of a man-like ape. Am. J. phys. Anthrop., (N.S.) 2: 1-129.

- 1956. Postembryonic age changes. In: H. HofER \& A. H. Schultz eds., Primatologia, 1: $887-964$ (Karger, Basel).

SteINER, H., 1949. Beobachtungen über das brutbiologische Verhalten des Gibbons und an einem frühgeborenen Fötus einer Bastardzucht von Hylobates. Arch. Julius Klaus-Stift. Vererb.-Forsch., 24: 217-236.

Voss, G., 1970. On the gestation of the White-handed Gibbon or Lar, Hylobates lar L.. Zool. Gart. Lpz., (N.F.) $39(1 / 6)$ : 295-296.

Welt, C. \& W. J. Welker, 1963. Postural and locomotor development of a gibbon (Hylobates lar). Am. J. phys. Anthrop., (N.S.) 21: 425-437.

Yerkes, R. M. \& A. W. Yerkes, 1929. The great apes. A study of anthropoid life: i-xix, 1-652 (Yale Univ. Press, New Haven; Humphrey Milford, London; Univ. Press, Oxford). 